\title{
Certain Integrals Associated with Hypergeometric Functions of Four Variables
}

\author{
Maged G. Bin-Saad $^{1}$ and Jihad A. Younis ${ }^{2}$ \\ ${ }^{1}$ Department of Mathematics, Aden University, Aden, Khormaksar, P.O. Box 6014, Yemen \\ e-mail:mgbinsaad@yahoo.com \\ ${ }^{2}$ Department of Mathematics, Aden University, Aden, Khormaksar, P.O. Box 6014, Yemen \\ e-mail: jihadalsaqqaf@gmail.com
}

\begin{abstract}
The main objective of this paper is to present integral representations of Euler type and Laplace type for five new hypergeometric series of four variables.
\end{abstract}

\section{Introduction}

In diverse areas in engineering and mathematical physics, integral representations play an important role in the view point of application. A number of integral representations involving various hypergeometric functions have been investigated by many authors (see [1, 2, 3, 4, 5, 6, 8, 15, 17]). Very recently, Bin-Saad et al. [2, 3] have introduced and studied ten quadruple hypergeometric functions $X_{1}^{(4)}, X_{2}^{(4)}, \ldots, X_{10}^{(4)}$. In [17] Younis and Bin-Saad gave integral representations for twenty new hypergeometric functions of four variables $X_{31}^{(4)}, X_{32}^{(4)}, \ldots, X_{50}^{(4)}$. Motivated mainly by the works [2, 3, 17], we further derive the following five new hypergeometric functions of four variables:

Received: May 18, 2019; Accepted: July 7, 2019

2010 Mathematics Subject Classification: Primary 33C20; Secondary 33C65.

Keywords and phrases: Gauss hypergeometric function, triple hypergeometric functions, integrals of Euler type, Laplace integral, Exton's hypergeometric functions, Appell functions, confluent hypergeometric functions.

Copyright (C) 2019 Maged G. Bin-Saad and Jihad A. Younis. This is an open access article distributed under the Creative Commons Attribution License, which permits unrestricted use, distribution, and reproduction in any medium, provided the original work is properly cited. 


$$
\begin{aligned}
& X_{21}^{(4)}\left(a_{1}, a_{1}, a_{2}, a_{1}, a_{1}, a_{2}, a_{3}, a_{2} ; c_{1}, c_{1}, c_{2}, c_{3} ; x, y, z, u\right) \\
& =\sum_{m, n, p, q=0}^{\infty} \frac{\left(a_{1}\right)_{2 m+n+q}\left(a_{2}\right)_{q+n+p}\left(a_{3}\right)_{p}}{\left(c_{1}\right)_{m+n}\left(c_{2}\right)_{p}\left(c_{3}\right)_{q}} \frac{x^{m}}{m !} \frac{y^{n}}{n !} \frac{z^{p}}{p !} \frac{u^{q}}{q !} \\
& X_{22}^{(4)}\left(a_{1}, a_{1}, a_{2}, a_{1}, a_{1}, a_{2}, a_{3}, a_{2} ; c_{2}, c_{1}, c_{1}, c_{3} ; x, y, z, u\right) \\
& =\sum_{m, n, p, q=0}^{\infty} \frac{\left(a_{1}\right)_{2 m+n+q}\left(a_{2}\right)_{q+n+p}\left(a_{3}\right)_{p}}{\left(c_{1}\right)_{n+p}\left(c_{2}\right)_{m}\left(c_{3}\right)_{q}} \frac{x^{m}}{m !} \frac{y^{n}}{n !} \frac{z^{p}}{p !} \frac{u^{q}}{q !}, \\
& X_{23}^{(4)}\left(a_{1}, a_{1}, a_{2}, a_{1}, a_{1}, a_{2}, a_{3}, a_{2} ; c_{1}, c_{1}, c_{1}, c_{2} ; x, y, z, u\right) \\
& =\sum_{m, n, p, q=0}^{\infty} \frac{\left(a_{1}\right)_{2 m+n+q}\left(a_{2}\right)_{q+n+p}\left(a_{3}\right)_{p}}{\left(c_{1}\right)_{m+n+p}\left(c_{2}\right)_{q}} \frac{x^{m}}{m !} \frac{y^{n}}{n !} \frac{z^{p}}{p !} \frac{u^{q}}{q !}, \\
& X_{24}^{(4)}\left(a_{1}, a_{1}, a_{2}, a_{1}, a_{1}, a_{2}, a_{3}, a_{3} ; c_{1}, c_{2}, c_{1}, c_{3} ; x, y, z, u\right) \\
& =\sum_{m, n, p, q=0}^{\infty} \frac{\left(a_{1}\right)_{2 m+n+q}\left(a_{2}\right)_{n+p}\left(a_{3}\right)_{p+q}}{\left(c_{1}\right)_{m+p}\left(c_{2}\right)_{n}\left(a_{3}\right)_{q}} \frac{x^{m}}{m !} \frac{y^{n}}{n !} \frac{z^{p}}{p !} \frac{u^{q}}{q !}, \\
& X_{25}^{(4)}\left(a_{1}, a_{1}, a_{3}, a_{1}, a_{1}, a_{2}, a_{4}, a_{2} ; c_{1}, c_{2}, c_{1}, c_{3} ; x, y, z, u\right) \\
& =\sum_{m, n, p, q=0}^{\infty} \frac{\left(a_{1}\right)_{2 m+n+q}\left(a_{2}\right)_{n+q}\left(a_{3}\right)_{p}\left(a_{4}\right)_{p}}{\left(c_{1}\right)_{m+p}\left(c_{2}\right)_{n}\left(c_{3}\right)_{q}} \frac{x^{m}}{m !} \frac{y^{n}}{n !} \frac{z^{p}}{p !} \frac{u^{q}}{q !}
\end{aligned}
$$

where $(a)_{m}$ denotes the Pochhammer symbol given by

$$
(a)_{m}=\frac{\Gamma(a+m)}{\Gamma(a)}=a(a+1) \cdots(a+m-1) \quad(m \in N:=\{1,2,3\}) \text { and }(a)_{0}=1 .
$$

In the present paper, we will introduce and study several integral representations for the new quadruple functions defined above. In Section 2, we have obtained some Euler type integrals involving quadruple functions $X_{i}^{(4)}(i=21,22,23,24,25)$. Then we aim to present integral representations of Laplace-type are for each functions $X_{i}^{(4)}(i=21,22,23,24,25)$. 


\section{Integral Representations of Euler-Type}

Here, in order to present our main finding, we recall the definition of some wellknown hypergeometric functions as follows:

The Gaussian hypergeometric function is defined by [15]

$$
{ }_{2} F_{1}(a, b ; c ; x)=\sum_{n=0}^{\infty} \frac{(a)_{n}(b)_{n}}{(c)_{n}} \frac{x^{n}}{n !}, \quad(|x|<1) .
$$

Appell's hypergeometric functions $F_{2}, F_{3}$ and $F_{4}$ of two variables and the Horn's series $\mathrm{H}_{4}$ of two variables are given by

$$
\begin{gathered}
F_{2}(a, b, c ; d, e ; x, y)=\sum_{m, n=0}^{\infty} \frac{(a)_{m+n}(b)_{m}(c)_{n}}{(d)_{m}(e)_{n}} \frac{x^{m}}{m !} \frac{y^{n}}{n !}, \quad(|x|+|y|<1), \\
F_{3}(a, b, c, d ; e ; x, y)=\sum_{m, n=0}^{\infty} \frac{(a)_{m}(b)_{n}(c)_{m}(d)_{n}}{(e)_{m+n}} \frac{x^{m}}{m !} \frac{y^{n}}{n !}, \quad(\max \{|x|,|y|\}<1), \\
F_{4}(a, b ; c, d ; x, y)=\sum_{m, n=0}^{\infty} \frac{(a)_{m+n}(b)_{m+n}}{(c)_{m}(d)_{n}} \frac{x^{m}}{m !} \frac{y^{n}}{n !}, \quad(\sqrt{|x|}+\sqrt{|y|}<1)
\end{gathered}
$$

and

$$
H_{4}(a, b ; c, d ; x, y)=\sum_{m, n=0}^{\infty} \frac{(a)_{2 m+n}(b)_{n}}{(c)_{m}(d)_{n}} \frac{x^{m}}{m !} \frac{y^{n}}{n !}, \quad\left(|x|<r,|y|<s, 4 r=(s-1)^{2}\right)
$$

respectively (see [15]). The Exton's triple functions $X_{2}, X_{3}, X_{4}, X_{13}, X_{14}, X_{15}, X_{16}$ and $X_{20}[9]$ are defined by as follows:

$$
\begin{aligned}
& X_{2}(a, b ; c, d, e ; x, y, z)=\sum_{m, n, p=0}^{\infty} \frac{(a)_{2 m+2 n+p}(b)_{p}}{(c)_{m}(d)_{n}(e)_{p}} \frac{x^{m}}{m !} \frac{y^{n}}{n !} \frac{z^{p}}{p !}, \\
& X_{3}(a, b ; c, d ; x, y, z)=\sum_{m, n, p=0}^{\infty} \frac{(a)_{2 m+n+p}(b)_{n+p}}{(c)_{m+n}(d)_{p}} \frac{x^{m}}{m !} \frac{y^{n}}{n !} \frac{z^{p}}{p !},
\end{aligned}
$$




$$
\begin{aligned}
& X_{4}(a, b ; c, d, e ; x, y, z)=\sum_{m, n, p=0}^{\infty} \frac{(a)_{2 m+n+p}(b)_{n+p}}{(c)_{m}(d)_{n}(e)_{p}} \frac{x^{m}}{m !} \frac{y^{n}}{n !} \frac{z^{p}}{p !}, \\
& X_{13}(a, b, c ; d ; x, y, z)=\sum_{m, n, p=0}^{\infty} \frac{(a)_{2 m+n}(b)_{n+p}(c)_{p}}{(d)_{m+n+p}} \frac{x^{m}}{m !} \frac{y^{n}}{n !} \frac{z^{p}}{p !}, \\
& X_{14}(a, b, c ; d, e ; x, y, z)=\sum_{m, n, p=0}^{\infty} \frac{(a)_{2 m+n}(b)_{n+p}(c)_{p}}{(d)_{m+n}(e)_{p}} \frac{x^{m}}{m !} \frac{y^{n}}{n !} \frac{z^{p}}{p !}, \\
& X_{15}(a, b, c ; d, e ; x, y, z)=\sum_{m, n, p=0}^{\infty} \frac{(a)_{2 m+n}(b)_{n+p}(c)_{p}}{(d)_{m}(e)_{n+p}} \frac{x^{m}}{m !} \frac{y^{n}}{n !} \frac{z^{p}}{p !}, \\
& X_{16}(a, b, c ; d, e ; x, y, z)=\sum_{m, n, p=0}^{\infty} \frac{(a)_{2 m+n}(b)_{n+p}(c)_{p}}{(d)_{m+p}(e)_{n}} \frac{x^{m}}{m !} \frac{y^{n}}{n !} \frac{z^{p}}{p !}
\end{aligned}
$$

and

$$
X_{20}(a, b, c, d ; e, f ; x, y, z)=\sum_{m, n, p=0}^{\infty} \frac{(a)_{2 m+n}(b)_{n}(c)_{p}(d)_{p}}{(e)_{m+p}(f)_{n}} \frac{x^{m}}{m !} \frac{y^{n}}{n !} \frac{z^{p}}{p !}
$$

The Lauricella's triple functions $F_{C}^{(3)}$ and $F_{F}$ (see [11]) are given by

$$
F_{C}^{(3)}\left(a, b ; c_{1}, c_{2}, c_{3} ; x, y, z\right)=\sum_{m, n, p=0}^{\infty} \frac{(a)_{m+n+p}(b)_{m+n+p}}{\left(c_{1}\right)_{m}\left(c_{2}\right)_{n}\left(c_{3}\right)_{p}} \frac{x^{m}}{m !} \frac{y^{n}}{n !} \frac{z^{p}}{p !}
$$

and

$$
F_{F}(a, a, a, b, c, b ; d, e, e ; x, y, z)=\sum_{m, n, p=0}^{\infty} \frac{(a)_{m+n+p}(b)_{m+p}(c)_{n}}{(d)_{m}(e)_{n+p}} \frac{x^{m}}{m !} \frac{y^{n}}{n !} \frac{z^{p}}{p !}
$$

Sharma and Parihar hypergeometric function of four variables $F_{14}^{(4)}$ is as follows (see [12]): 


$$
\begin{aligned}
& F_{14}^{(4)}\left(a_{1}, a_{1}, a_{1}, a_{2}, b_{1}, b_{1}, b_{1}, b_{2} ; c_{1}, c_{2}, c_{3}, c_{1} ; x, y, z, u\right) \\
= & \sum_{m, n, p, q=0}^{\infty} \frac{\left(a_{1}\right)_{m+n+p}\left(a_{2}\right)_{q}\left(b_{1}\right)_{m+n+p}\left(b_{2}\right)_{q}}{\left(c_{1}\right)_{m+q}\left(c_{2}\right)_{n}\left(c_{3}\right)_{p}} \frac{x^{m}}{m !} \frac{y^{n}}{n !} \frac{z^{p}}{p !} \frac{u^{q}}{q !} .
\end{aligned}
$$

Lauricella hypergeometric function of four variables $F_{C}^{(4)}$ is as below (see [10])

$$
\begin{aligned}
& F_{C}^{(4)}\left(a, b ; c_{1}, c_{2}, c_{3}, c_{4} ; x, y, z, u\right) \\
&= \sum_{m, n, p, q=0}^{\infty} \frac{(a)_{m+n+p+q}(b)_{m+n+p+q}}{\left(c_{1}\right)_{m}\left(c_{2}\right)_{n}\left(c_{3}\right)_{p}\left(c_{4}\right)_{q}} \frac{x^{m}}{m !} \frac{y^{n}}{n !} \frac{z^{p}}{p !} \frac{u^{q}}{q !} \\
&(\sqrt{|x|}+\sqrt{|y|}+\sqrt{|z|}+\sqrt{|u|}<1) .
\end{aligned}
$$

Now, we evaluate five integrals representations for each functions $X_{i}^{(4)}$ $(i=21,22,23,24,25)$ involving the Gauss hypergeometric function ${ }_{2} F_{1}$, Appell hypergeometric functions $F_{2}, F_{3}$ and $F_{4}$, Horn's function $H_{4}$ of two variables, the Exton's triple series $X_{2}, X_{3}, X_{4}, X_{13}, X_{14}, X_{15}, X_{16}$ and $X_{20}$, the Lauricella's triple series $F_{C}^{(3)}$ and $F_{F}$, and the quadruple series $F_{14}^{(4)}, X_{21}^{(4)}$ and $F_{C}^{(4)}$, as follows:

$$
\begin{aligned}
& X_{21}^{(4)}\left(a_{1}, a_{1}, a_{2}, a_{1}, a_{1}, a_{2}, a_{3}, a_{2} ; c_{1}, c_{1}, c_{2}, c_{3} ; x, y, z, u\right) \\
= & \frac{\Gamma\left(a_{1}+a_{2}+a_{3}\right) \Gamma\left(c_{1}\right)}{\Gamma\left(a_{1}\right) \Gamma\left(a_{2}\right) \Gamma\left(a_{3}\right) \Gamma(a) \Gamma\left(c_{1}-a\right)} \\
& \times \int_{0}^{1} \int_{0}^{1} \int_{0}^{1} \alpha^{a_{1}-1}(1-\alpha)^{a_{2}-1} \beta^{a_{1}+a_{2}-1}(1-\beta)^{a_{3}-1} \gamma^{a-1}(1-\gamma)^{c_{1}-a-1} \\
& \times F_{C}^{(4)}\left(\frac{a_{1}+a_{2}+a_{3}}{2}, \frac{a_{1}+a_{2}+a_{3}+1}{2} ; a, c_{1}-a, c_{2}, c_{3} ; \lambda_{1} x, \lambda_{2} y, \lambda_{3} z, \lambda_{4} u\right) d \alpha d \beta d \gamma \\
\left(\lambda_{1}=4 \alpha^{2} \beta^{2} \gamma, \lambda_{2}=4 \alpha(1-\alpha) \beta^{2}(1-\gamma), \lambda_{3}=4(1-\alpha) \beta(1-\beta), \lambda_{4}=4 \alpha(1-\alpha) \beta^{2}\right), & \left(\operatorname{Re}\left(a_{i}\right)>0, i=(1,2,3), \operatorname{Re}(a)>0, \operatorname{Re}\left(c_{1}-a\right)>0\right) ;
\end{aligned}
$$




$$
\begin{aligned}
& X_{21}^{(4)}\left(a_{1}, a_{1}, a_{2}, a_{1}, a_{1}, a_{2}, a_{3}, a_{2} ; c_{1}, c_{1}, c_{2}, c_{3} ; x, y, z, u\right) \\
& =\frac{\Gamma\left(a_{1}+a_{2}\right)}{\Gamma\left(a_{1}\right) \Gamma\left(a_{2}\right)} \int_{0}^{1} \alpha^{a_{1}-1}(1-\alpha)^{a_{2}-1} \\
& \times X_{2}\left(a_{1}+a_{2}, a_{3} ; c_{1}, c_{3}, c_{2} ; \alpha^{2} x+\alpha(1-\alpha) y, \alpha(1-\alpha) u,(1-\alpha) z\right) d \alpha \\
& \left(\operatorname{Re}\left(a_{1}\right)>0, \operatorname{Re}\left(a_{2}\right)>0\right) \\
& X_{21}^{(4)}\left(a_{1}, a_{1}, a_{2}, a_{1}, a_{1}, a_{2}, a_{3}, a_{2} ; c_{1}, c_{1}, c_{2}, c_{3} ; x, y, z, u\right) \\
& =\frac{2 \Gamma\left(c_{3}\right)}{\Gamma\left(a_{1}\right) \Gamma\left(c_{3}-a_{1}\right)} \int_{0}^{\frac{\pi}{2}}\left(\sin ^{2} \alpha\right)^{a_{1}-\frac{1}{2}}\left(\cos ^{2} \alpha\right)^{c_{3}-a_{1}-\frac{1}{2}}\left(1-u \sin ^{2} \alpha\right)^{-a_{2}} \\
& \times X_{14}\left(1+a_{1}-c_{3}, a_{2}, a_{3} ; c_{1}, c_{2} ; x \tan ^{4} \alpha,-\frac{y \tan ^{2} \alpha}{\left(1-u \sin ^{2} \alpha\right)}, \frac{z}{\left(1-u \sin ^{2} \alpha\right)}\right) d \alpha \\
& \left(\operatorname{Re}\left(a_{1}\right)>0, \operatorname{Re}\left(c_{3}-a_{1}\right)>0\right)
\end{aligned}
$$

$X_{21}^{(4)}\left(a_{1}, a_{1}, a_{2}, a_{1}, a_{1}, a_{2}, a_{3}, a_{2} ; c_{1}, c_{1}, c_{2}, c_{3} ; x, y, z, u\right)$

$$
\begin{aligned}
= & \frac{\Gamma\left(c_{1}\right)(S-T)^{a_{1}}(R-T)}{\Gamma\left(a_{1}\right) \Gamma\left(c_{1}-a_{1}\right)(S-R)^{2 c_{1}-a_{1}-a_{2}-2}} \int_{R}^{S}(\alpha-R)^{a_{1}-1}(\alpha-T)^{1+a_{1}+a_{2}-2 c_{1}} \\
& \times\left[(R-T)(S-R)(S-\alpha)(\alpha-T)+(S-T)^{2}(\alpha-R)^{2} x\right]^{c_{1}-a_{1}-1} \\
& \times[(S-R)(\alpha-T)-(S-T)(\alpha-R) y]^{-a_{1}} \\
& \times F_{2}\left(a_{2}, a_{3}, 1+a_{1}-c_{1} ; c_{2}, c_{3} ; \lambda_{1} z, \lambda_{2} u\right) d \alpha
\end{aligned}
$$

$$
\left.\begin{array}{c}
\left(\lambda_{1}=\frac{(S-R)(\alpha-T)}{[(S-R)(\alpha-T)-(S-T)(\alpha-R) y]},\right. \\
\left.\lambda_{2}=-\frac{(S-R)^{2}(S-T)(\alpha-R)(\alpha-T)^{2}}{\left[(R-T)(S-R)(S-\alpha)(\alpha-T)+(S-T)^{2}(\alpha-R)^{2} x\right]}\right), \\
\times[(S-R)(\alpha-T)-(S-T)(\alpha-R) y]
\end{array}\right),
$$


$X_{21}^{(4)}\left(a_{1}, a_{1}, a_{2}, a_{1}, a_{1}, a_{2}, a_{3}, a_{2} ; c_{1}, c_{1}, c_{2}, c_{3} ; x, y, z, u\right)$

$$
\begin{aligned}
= & \frac{\Gamma\left(c_{1}\right) \Gamma\left(c_{2}\right)}{\Gamma\left(a_{1}\right) \Gamma\left(a_{3}\right) \Gamma\left(c_{1}-a_{1}\right) \Gamma\left(c_{2}-a_{3}\right)} \\
& \times \int_{0}^{\infty} \int_{0}^{\infty}\left(e^{-\alpha}\right)^{a_{1}}\left(e^{-\beta}\right)^{a_{3}}\left(1-e^{-\beta}\right)^{c_{2}-a_{3}-1} \\
& \times\left[\left(1-e^{-\alpha}\right)+x e^{-2 \alpha}\right]^{c_{1}-a_{1}-1}\left(1-y e^{-\alpha}-z e^{-\beta}\right)^{-a_{2}} \\
& \times{ }_{2} F_{1}\left(1+a_{1}-c_{1}, a_{2} ; c_{3} ;-\frac{u e^{-\alpha}}{\left[\left(1-e^{-\alpha}\right)+x e^{-2 \alpha}\right]\left(1-y e^{-\alpha}-z e^{-\beta}\right)}\right) d \alpha d \beta \\
& \left(\operatorname{Re}\left(a_{1}\right)>0, \operatorname{Re}\left(a_{3}\right)>0, \operatorname{Re}\left(c_{1}-a_{1}\right)>0, \operatorname{Re}\left(c_{2}-a_{3}\right)>0\right) ;
\end{aligned}
$$

$X_{22}^{(4)}\left(a_{1}, a_{1}, a_{2}, a_{1}, a_{1}, a_{2}, a_{3}, a_{2} ; c_{2}, c_{1}, c_{1}, c_{3} ; x, y, z, u\right)$

$$
=\frac{\Gamma\left(a_{1}+a_{3}\right)}{\Gamma\left(a_{1}\right) \Gamma\left(a_{3}\right)} \int_{0}^{\infty}\left(e^{-\alpha}\right)^{a_{1}}\left(1-e^{-\alpha}\right)^{a_{3}-1}
$$$$
\times X_{4}\left(a_{1}+a_{3}, a_{2} ; c_{2}, c_{1}, c_{3} ; x e^{-2 \alpha}, y e^{-\alpha}+z\left(1-e^{-\alpha}\right), u e^{-\alpha}\right) d \alpha
$$

$$
\left(\operatorname{Re}\left(a_{1}\right)>0, \operatorname{Re}\left(a_{3}\right)>0\right)
$$

$$
X_{22}^{(4)}\left(a_{1}, a_{1}, a_{2}, a_{1}, a_{1}, a_{2}, a_{3}, a_{2} ; c_{2}, c_{1}, c_{1}, c_{3} ; x, y, z, u\right)
$$

$$
=\frac{\Gamma\left(c_{3}\right)}{\Gamma\left(a_{1}\right) \Gamma\left(c_{3}-a_{1}\right)} \int_{0}^{1} \alpha^{a_{1}-1}(1-\alpha)^{c_{3}-a_{1}-1}(1-\alpha u)^{-a_{2}}
$$

$$
\begin{gathered}
\times X_{15}\left(1+a_{1}-c_{3}, a_{2}, a_{3} ; c_{2}, c_{1} ; \frac{\alpha^{2} x}{(1-\alpha)^{2}}, \frac{\alpha y}{(1-\alpha)(1-\alpha u)}, \frac{z}{(1-\alpha u)}\right) d \alpha \\
\left(\operatorname{Re}\left(a_{1}\right)>0, \operatorname{Re}\left(c_{3}-a_{1}\right)>0\right) ;
\end{gathered}
$$

$X_{22}^{(4)}\left(a_{1}, a_{1}, a_{2}, a_{1}, a_{1}, a_{2}, a_{3}, a_{2} ; c_{2}, c_{1}, c_{1}, c_{3} ; x, y, z, u\right)$

$=\frac{\Gamma\left(c_{2}\right)}{\Gamma\left(a_{1}\right) \Gamma\left(c_{2}-a_{1}\right)} \int_{0}^{\infty} \alpha^{a_{1}-1}(1+\alpha)^{1+a_{1}-2 c_{2}}\left[(1+\alpha)+\alpha^{2} x\right]^{c_{2}-a_{1}-1}$ 
$\times F_{F}\left(a_{2}, a_{2}, a_{2}, 1+a_{1}-c_{2}, a_{3}, 1+a_{1}-c_{2} ; c_{3}, c_{1}, c_{1} ; \lambda u, z, \lambda y\right) d \alpha$

$$
\begin{gathered}
\left(\lambda=-\frac{\alpha(1+\alpha)}{\left[(1+\alpha)+\alpha^{2} x\right]}\right), \\
\left(\operatorname{Re}\left(a_{1}\right)>0, \operatorname{Re}\left(c_{2}-a_{1}\right)>0\right) ;
\end{gathered}
$$

$X_{22}^{(4)}\left(a_{1}, a_{1}, a_{2}, a_{1}, a_{1}, a_{2}, a_{3}, a_{2} ; c_{2}, c_{1}, c_{1}, c_{3} ; x, y, z, u\right)$

$$
\begin{aligned}
& =\frac{2 \Gamma\left(c_{1}\right)}{\Gamma\left(a_{2}\right) \Gamma\left(c_{1}-a_{2}\right)} \\
& \times \int_{0}^{\frac{\pi}{2}}\left(\sin ^{2} \alpha\right)^{a_{2}-\frac{1}{2}}\left(\cos ^{2} \alpha\right)^{c_{1}-a_{2}-\frac{1}{2}}\left(1-y \sin ^{2} \alpha\right)^{-a_{1}}\left(1-z \sin ^{2} \alpha\right)^{-a_{3}} \\
& \times H_{4}\left(a_{1}, 1+a_{2}-c_{1} ; c_{2}, c_{3} ; \frac{x}{\left(1-y \sin ^{2} \alpha\right)^{2}},-\frac{u \tan ^{2} \alpha}{\left(1-y \sin ^{2} \alpha\right)}\right) d \alpha \\
& \left.\qquad \operatorname{Re}\left(a_{2}\right)>0, \operatorname{Re}\left(c_{1}-a_{2}\right)>0\right) ;
\end{aligned}
$$

$X_{22}^{(4)}\left(a_{1}, a_{1}, a_{2}, a_{1}, a_{1}, a_{2}, a_{3}, a_{2} ; c_{2}, c_{1}, c_{1}, c_{3} ; x, y, z, u\right)$

$$
\begin{aligned}
& =\frac{4 \Gamma\left(c_{1}\right) \Gamma\left(c_{2}\right)}{\Gamma\left(a_{1}\right) \Gamma\left(a_{2}\right) \Gamma\left(c_{1}-a_{2}\right) \Gamma\left(c_{2}-a_{1}\right)} \\
& \times \int_{0}^{\frac{\pi}{2}} \int_{0}^{\frac{\pi}{2}}\left(\sin ^{2} \alpha\right)^{a_{1}-\frac{1}{2}}\left(\cos ^{2} \alpha\right)^{c_{2}-a_{1}-\frac{1}{2}}\left(\sin ^{2} \beta\right)^{a_{2}-\frac{1}{2}}\left(\cos ^{2} \beta\right)^{c_{1}-a_{2}-\frac{1}{2}} \\
& \times\left(1+x \sin ^{2} \alpha \tan ^{2} \alpha+y \tan ^{2} \alpha \sin ^{2} \beta\right)^{c_{2}-a_{1}-1}\left(1-z \sin ^{2} \beta\right)^{-a_{3}} \\
& \times_{2} F_{1}\left(1+a_{1}-c_{2}, 1+a_{2}-c_{1} ; c_{3} ; \frac{u \tan ^{2} \alpha \tan ^{2} \beta}{\left(1+x \sin ^{2} \alpha \tan ^{2} \alpha+y \tan ^{2} \alpha \sin ^{2} \beta\right)}\right) d \alpha d \beta \\
& \left(\operatorname{Re}\left(a_{1}\right)>0, \operatorname{Re}\left(a_{2}\right)>0, \operatorname{Re}\left(c_{1}-a_{2}\right)>0, \operatorname{Re}\left(c_{2}-a_{1}\right)>0\right) ; \\
& X_{23}^{(4)}\left(a_{1}, a_{1}, a_{2}, a_{1}, a_{1}, a_{2}, a_{3}, a_{2} ; c_{1}, c_{1}, c_{1}, c_{2} ; x, y, z, u\right) \\
& =\frac{2 \Gamma\left(c_{1}\right)}{\Gamma(a) \Gamma\left(c_{1}-a\right)} \int_{0}^{\frac{\pi}{2}}\left(\sin ^{2} \alpha\right)^{a-\frac{1}{2}}\left(\cos ^{2} \alpha\right)^{c_{1}-a-\frac{1}{2}}
\end{aligned}
$$


$\times X_{21}^{(4)}\left(a_{1}, a_{1}, a_{2}, a_{1}, a_{1}, a_{2}, a_{3}, a_{2} ; a, a, c_{1}-a, c_{2}\right.$;

$$
\begin{aligned}
\left.x \sin ^{2} \alpha, y \sin ^{2} \alpha, z \cos ^{2} \alpha, u\right) d \alpha \\
\left(\operatorname{Re}(a)>0, \operatorname{Re}\left(c_{1}-a\right)>0\right) ;
\end{aligned}
$$

$X_{23}^{(4)}\left(a_{1}, a_{1}, a_{2}, a_{1}, a_{1}, a_{2}, a_{3}, a_{2} ; c_{1}, c_{1}, c_{1}, c_{2} ; x, y, z, u\right)$

$$
\begin{aligned}
= & \frac{\Gamma\left(c_{1}\right)(1+M)^{a_{3}}}{\Gamma\left(a_{3}\right) \Gamma\left(c_{1}-a_{3}\right)} \int_{0}^{1} \alpha^{a_{1}-1}(1-\alpha)^{c_{1}-a_{3}-1}(1+M \alpha)^{a_{2}-c_{1}}[(1+M \alpha)-(1+M) \alpha z]^{-a_{2}} \\
& \times X_{3}\left(a_{1}, a_{2} ; c_{1}-a_{3}, c_{2} ; \frac{(1-\alpha) x}{(1+M \alpha)}, \frac{(1-\alpha) y}{[(1+M \alpha)-(1+M) \alpha z]}, \frac{(1+M \alpha) u}{[(1+M \alpha)-(1+M) \alpha z]}\right) d \alpha
\end{aligned}
$$

$$
\left(\operatorname{Re}\left(a_{3}\right)>0, \operatorname{Re}\left(c_{1}-a_{3}\right)>0, M>-1\right)
$$

$X_{23}^{(4)}\left(a_{1}, a_{1}, a_{2}, a_{1}, a_{1}, a_{2}, a_{3}, a_{2} ; c_{1}, c_{1}, c_{1}, c_{2} ; x, y, z, u\right)$

$$
\begin{gathered}
=\frac{\Gamma\left(c_{2}\right)}{\Gamma\left(a_{2}\right) \Gamma\left(c_{2}-a_{2}\right)} \int_{0}^{\infty}\left(e^{-\alpha}\right)^{a_{2}}\left(1-e^{-\alpha}\right)^{c_{2}-a_{2}-1}\left(1-u e^{-\alpha}\right)^{-a_{1}} \\
\times X_{13}\left(a_{1}, 1+a_{2}-c_{2}, a_{3} ; c_{1} ; \frac{x}{\left(1-u e^{-\alpha}\right)^{2}},-\frac{y e^{-\alpha}}{\left(1-e^{-\alpha}\right)\left(1-u e^{-\alpha}\right)},-\frac{z e^{-\alpha}}{\left(1-e^{-\alpha}\right)}\right) d \alpha \\
\left(\operatorname{Re}\left(a_{2}\right)>0, \operatorname{Re}\left(c_{2}-a_{2}\right)>0\right) ;
\end{gathered}
$$

$X_{23}^{(4)}\left(a_{1}, a_{1}, a_{2}, a_{1}, a_{1}, a_{2}, a_{3}, a_{2} ; c_{1}, c_{1}, c_{1}, c_{2} ; x, y, z, u\right)$

$$
\begin{aligned}
& =\frac{2 \Gamma\left(c_{2}\right) M^{a_{1}}}{\Gamma\left(a_{1}\right) \Gamma\left(c_{2}-a_{1}\right)} \int_{0}^{\frac{\pi}{2}}\left(\sin ^{2} \alpha\right)^{a_{1}-\frac{1}{2}}\left(\cos ^{2} \alpha\right)^{c_{2}-a_{1}-\frac{1}{2}}\left(\cos ^{2} \alpha+M \sin ^{2} \alpha\right)^{a_{2}-c_{2}} \\
& \times\left[\left(\cos ^{2} \alpha+M \sin ^{2} \alpha\right)-M u \sin ^{2} \alpha\right]^{-a_{2}} \\
& \times X_{13}\left(1+a_{1}-c_{2}, a_{2}, a_{3} ; c_{1} ; M^{2} x \tan ^{4} \alpha, \lambda_{1} y, \lambda_{2} z\right) d \alpha \\
& \left(\lambda_{1}=-\frac{M\left(\cos ^{2} \alpha+M \sin ^{2} \alpha\right) \tan ^{2} \alpha}{\left[\left(\cos ^{2} \alpha+M \sin ^{2} \alpha\right)-M u \sin ^{2} \alpha\right]}, \lambda_{2}=\frac{\left(\cos ^{2} \alpha+M \sin ^{2} \alpha\right)}{\left[\left(\cos ^{2} \alpha+M \sin ^{2} \alpha\right)-M u \sin ^{2} \alpha\right]}\right), \\
& \left(\operatorname{Re}\left(a_{1}\right)>0, \operatorname{Re}\left(c_{2}-a_{1}\right)>0, M>0\right) ;
\end{aligned}
$$




$$
\begin{aligned}
& X_{23}^{(4)}\left(a_{1}, a_{1}, a_{2}, a_{1}, a_{1}, a_{2}, a_{3}, a_{2} ; c_{1}, c_{1}, c_{1}, c_{2} ; x, y, z, u\right) \\
&= \frac{\Gamma\left(a_{1}+a_{2}+a_{3}\right)}{\Gamma\left(a_{1}\right) \Gamma\left(a_{2}\right) \Gamma\left(a_{3}\right)} \int_{0}^{1} \int_{0}^{1} \alpha^{a_{1}-1}(1-\alpha)^{a_{2}-1} \beta^{a_{1}+a_{2}-1}(1-\beta)^{a_{3}-1} \\
& \times F_{4}\left(\frac{a_{1}+a_{2}+a_{3}}{2}, \frac{a_{1}+a_{2}+a_{3}+1}{2}, c_{1}, c_{2} ;\right. \\
&\left.4 \alpha^{2} \beta^{2} x+4 \alpha(1-\alpha) \beta^{2} y+4(1-\alpha) \beta(1-\beta) z, 4 \alpha(1-\alpha) \beta^{2} u\right) d \alpha d \beta \\
&\left(\operatorname{Re}\left(a_{1}\right)>0, i=(1,2,3)\right) ;
\end{aligned}
$$$$
X_{24}^{(4)}\left(a_{1}, a_{1}, a_{2}, a_{1}, a_{1}, a_{2}, a_{3}, a_{3} ; c_{1}, c_{2}, c_{1}, c_{3} ; x, y, z, u\right)
$$$$
=\frac{2 \Gamma\left(c_{3}\right)}{\Gamma\left(a_{1}\right) \Gamma\left(c_{3}-a_{1}\right)} \int_{0}^{\frac{\pi}{2}}\left(\sin ^{2} \alpha\right)^{a_{1}-\frac{1}{2}}\left(\cos ^{2} \alpha\right)^{c_{3}-a_{1}-\frac{1}{2}}\left(1-u \sin ^{2} \alpha\right)^{-a_{3}}
$$$$
\times X_{16}\left(1+a_{1}-c_{3}, a_{2}, a_{3} ; c_{1}, c_{2} ; x \tan ^{4} \alpha,-y \tan ^{2} \alpha, \frac{z}{\left(1-u \sin ^{2} \alpha\right)}\right) d \alpha
$$$$
\left(\operatorname{Re}\left(a_{1}\right)>0, \operatorname{Re}\left(c_{3}-a_{1}\right)>0\right)
$$

$X_{24}^{(4)}\left(a_{1}, a_{1}, a_{2}, a_{1}, a_{1}, a_{2}, a_{3}, a_{3} ; c_{1}, c_{2}, c_{1}, c_{3} ; x, y, z, u\right)$$$
=\frac{\Gamma\left(c_{3}\right)(S-T)^{a_{3}}(R-T)^{c_{3}-a_{3}}}{\Gamma\left(a_{3}\right) \Gamma\left(c_{3}-a_{3}\right)(S-R)^{c_{3}-a_{1}-1}}
$$$$
\times \int_{R}^{S}(\alpha-R)^{a_{3}-1}(S-\alpha)^{c_{3}-a_{3}-1}(\alpha-T)^{a_{1}-c_{3}}[(S-R)(\alpha-T)-(S-T)(\alpha-R) u]^{-a_{1}}
$$$$
\times X_{16}\left(a_{1}, a_{2}, 1+a_{3}-c_{3} ; c_{1}, c_{2} ; \lambda_{1} x, \lambda_{2} y, \lambda_{3} z\right) d \alpha
$$

$$
\begin{aligned}
\left(\lambda_{1}\right. & =\frac{(S-R)^{2}(\alpha-T)^{2}}{[(S-R)(\alpha-T)-(S-T)(\alpha-R) u]^{2}}, \lambda_{2}=\frac{(S-R)(\alpha-T)}{[(S-R)(\alpha-T)-(S-T)(\alpha-R) u]}, \\
\lambda_{3} & \left.=-\frac{(R-T)(S-\alpha)}{(S-R)(\alpha-T)}\right),
\end{aligned}
$$

$$
\left(\operatorname{Re}\left(a_{3}\right)>0, \operatorname{Re}\left(c_{3}-a_{3}\right)>0, T<R<S\right) ;
$$




$$
\begin{gathered}
X_{24}^{(4)}\left(a_{1}, a_{1}, a_{2}, a_{1}, a_{1}, a_{2}, a_{3}, a_{3} ; c_{1}, c_{2}, c_{1}, c_{3} ; x, y, z, u\right) \\
=\frac{\Gamma\left(a_{1}+a_{2}+a_{3}\right)}{\Gamma\left(a_{1}\right) \Gamma\left(a_{2}\right) \Gamma\left(a_{3}\right)} \int_{0}^{\infty} \int_{0}^{\infty}\left(e^{-\alpha}\right)^{a_{1}}\left(1-e^{-\alpha}\right)^{a_{2}-1}\left(e^{-\beta}\right)^{a_{1}+a_{2}}\left(1-e^{-\beta}\right)^{a_{3}-1} \\
\times F_{C}^{(3)}\left(\frac{a_{1}+a_{2}+a_{3}}{2}, \frac{a_{1}+a_{2}+a_{3}+1}{2} ; c_{1}, c_{2}, c_{3} ; \lambda_{1} x+\lambda_{2} z, \lambda_{3} y, \lambda_{4} u\right) d \alpha d \beta \\
\left(\lambda_{1}=4 e^{-2(\alpha+\beta)}, \lambda_{2}=4\left(1-e^{-\alpha}\right) e^{-\beta}\left(1-e^{-\beta}\right),\right. \\
\left.\lambda_{3}=4 e^{-(\alpha+2 \beta)}\left(1-e^{-\alpha}\right), \lambda_{4}=4 e^{-(\alpha+\beta)}\left(1-e^{-\beta}\right)\right), \\
\left(\operatorname{Re}\left(a_{i}\right)>0, i=(1,2,3)\right) ;
\end{gathered}
$$

$X_{24}^{(4)}\left(a_{1}, a_{1}, a_{2}, a_{1}, a_{1}, a_{2}, a_{3}, a_{3} ; c_{1}, c_{2}, c_{1}, c_{3} ; x, y, z, u\right)$

$$
\begin{aligned}
& =\frac{\Gamma\left(c_{2}\right) \Gamma\left(c_{3}\right)}{\Gamma\left(a_{2}\right) \Gamma\left(a_{3}\right) \Gamma\left(c_{2}-a_{2}\right) \Gamma\left(c_{3}-a_{3}\right)} \\
& \quad \times \int_{0}^{1} \int_{0}^{1} \alpha^{a_{2}-1}(1-\alpha)^{c_{2}-a_{2}-1} \beta^{a_{3}-1}(1-\beta)^{c_{3}-a_{3}-1}(1-\alpha y-\beta u)^{-a_{1}} \\
& \quad \times F_{3}\left(\frac{a_{1}}{2}, 1+a_{2}-c_{2}, \frac{a_{1}+1}{2}, 1+a_{3}-c_{3} ; c_{1} ; \frac{4 x}{(1-\alpha y-\beta u)^{2}}, \frac{\alpha \beta z}{(1-\alpha)(1-\beta)}\right) d \alpha d \beta \\
& \quad\left(\operatorname{Re}\left(a_{2}\right)>0, \operatorname{Re}\left(a_{3}\right)>0, \operatorname{Re}\left(c_{2}-a_{2}\right)>0, \operatorname{Re}\left(c_{3}-a_{3}\right)>0\right) ;
\end{aligned}
$$

$X_{24}^{(4)}\left(a_{1}, a_{1}, a_{2}, a_{1}, a_{1}, a_{2}, a_{3}, a_{3} ; c_{1}, c_{2}, c_{1}, c_{3} ; x, y, z, u\right)$

$$
\begin{aligned}
= & \frac{\Gamma\left(c_{2}\right) \Gamma\left(c_{3}\right)}{\Gamma\left(a_{2}\right) \Gamma\left(a_{3}\right) \Gamma\left(c_{2}-a_{2}\right) \Gamma\left(c_{3}-a_{3}\right)} \\
& \times \int_{0}^{\infty} \int_{0}^{\infty} \alpha^{a_{2}-1}(1+\alpha)^{a_{1}-c_{2}} \beta^{a_{3}-1}(1+\beta)^{a_{1}-c_{3}}[(1+\alpha)(1+\beta)-\alpha(1+\beta) y-(1+\alpha) \beta u]^{-a_{1}}
\end{aligned}
$$$$
\times F_{3}\left(\frac{a_{1}}{2}, 1+a_{2}-c_{2}, \frac{a_{1}+1}{2}, 1+a_{3}-c_{3} ; c_{1} ; \frac{4(1+\alpha)^{2}(1+\beta)^{2} x}{[(1+\alpha)(1+\beta)-\alpha(1+\beta) y-(1+\alpha) \beta u]^{2}}, \alpha \beta z\right)
$$

$\times d \alpha d \beta$

$$
\left(\operatorname{Re}\left(a_{2}\right)>0, \operatorname{Re}\left(a_{3}\right)>0, \operatorname{Re}\left(c_{2}-a_{2}\right)>0, \operatorname{Re}\left(c_{3}-a_{3}\right)>0\right) ;
$$




$$
\begin{aligned}
& X_{25}^{(4)}\left(a_{1}, a_{1}, a_{3}, a_{1}, a_{1}, a_{2}, a_{4}, a_{2} ; c_{1}, c_{2}, c_{1}, c_{3} ; x, y, z, u\right) \\
&= \frac{2 \Gamma\left(a_{1}+a_{2}\right)}{\Gamma\left(a_{1}\right) \Gamma\left(a_{2}\right)} \int_{0}^{\frac{\pi}{2}}\left(\sin ^{2} \alpha\right)^{a_{1}-\frac{1}{2}}\left(\cos ^{2} \alpha\right)^{a_{2}-\frac{1}{2}} \\
& \times F_{14}^{(4)}\left(\frac{a_{1}+a_{2}}{2}, \frac{a_{1}+a_{2}}{2}, \frac{a_{1}+a_{2}}{2}, a_{3}, \frac{a_{1}+a_{2}+1}{2},\right. \\
&\left.\frac{a_{1}+a_{2}+1}{2}, \frac{a_{1}+a_{2}+1}{2}, a_{4} ; c_{1}, c_{2}, c_{3}, c_{1} ; \lambda_{1} x, \lambda_{2} y, \lambda_{2} u, z\right) d \alpha \\
&\left(\lambda_{1}=4 \sin ^{4} \alpha, \lambda_{2}=\sin ^{2} 2 \alpha\right), \\
&\left(\operatorname{Re}\left(a_{1}\right)>0, \operatorname{Re}\left(a_{2}\right)>0\right) ;
\end{aligned}
$$$$
X_{25}^{(4)}\left(a_{1}, a_{1}, a_{3}, a_{1}, a_{1}, a_{2}, a_{4}, a_{2} ; c_{1}, c_{2}, c_{1}, c_{3} ; x, y, z, u\right)
$$$$
=\frac{\Gamma\left(c_{1}\right)(S-T)^{a_{3}}(R-T)^{c_{1}-a_{3}}}{\Gamma\left(a_{3}\right) \Gamma\left(c_{1}-a_{3}\right)(S-R)^{c_{1}-a_{4}-1}}
$$$$
\times \int_{R}^{S}(\alpha-R)^{a_{3}-1}(S-\alpha)^{c_{1}-a_{3}-1}(\alpha-T)^{a_{4}-c_{1}}[(S-R)(\alpha-T)-(S-T)(\alpha-R) z]^{-a_{4}}
$$$$
\times X_{4}\left(a_{1}, a_{2} ; c_{1}-a_{3}, c_{2}, c_{3} ; \frac{(R-T)(S-\alpha) x}{(S-R)(\alpha-T)}, y, u\right) d \alpha
$$$$
\left(\operatorname{Re}\left(a_{3}\right)>0, \operatorname{Re}\left(c_{1}-a_{3}\right)>0, T<R<S\right)
$$

$X_{25}^{(4)}\left(a_{1}, a_{1}, a_{3}, a_{1}, a_{1}, a_{2}, a_{4}, a_{2} ; c_{1}, c_{2}, c_{1}, c_{3} ; x, y, z, u\right)$

$$
\begin{gathered}
=\frac{\Gamma\left(c_{3}\right)}{\Gamma\left(a_{2}\right) \Gamma\left(c_{3}-a_{2}\right)} \int_{0}^{\infty} \alpha^{a_{2}-1}(1+\alpha)^{a_{1}-c_{3}}[(1+\alpha)-\alpha u]^{-a_{1}} \\
\times X_{20}\left(a_{1}, 1+a_{2}-c_{3}, a_{3}, a_{4} ; c_{1}, c_{2} ; \frac{(1+\alpha)^{2} x}{[(1+\alpha)-\alpha u]^{2}},-\frac{\alpha(1+\alpha) y}{[(1+\alpha)-\alpha u]}, z\right) d \alpha \\
\quad\left(\operatorname{Re}\left(a_{2}\right)>0, \operatorname{Re}\left(c_{3}-a_{2}\right)>0\right) ;
\end{gathered}
$$




$$
\begin{aligned}
& X_{25}^{(4)}\left(a_{1}, a_{1}, a_{3}, a_{1}, a_{1}, a_{2}, a_{4}, a_{2} ; c_{1}, c_{2}, c_{1}, c_{3} ; x, y, z, u\right) \\
& =\frac{\Gamma\left(a_{1}+a_{2}+a_{3}+a_{4}\right)}{\Gamma\left(a_{1}\right) \Gamma\left(a_{2}\right) \Gamma\left(a_{3}\right) \Gamma\left(a_{4}\right)} \\
& \times \int_{0}^{1} \int_{0}^{1} \int_{0}^{1} \alpha^{a_{1}-1}(1-\alpha)^{a_{2}-1} \beta^{a_{1}+a_{2}-1}(1-\beta)^{a_{3}-1} \gamma^{a_{1}+a_{2}+a_{3}-1}(1-\gamma)^{a_{4}-1} \\
& \times F_{C}^{(3)}\left(\frac{a_{1}+a_{2}+a_{3}+a_{4}}{2}, \frac{a_{1}+a_{2}+a_{3}+a_{4}+1}{2} ; c_{1}, c_{2}, c_{3} ; \lambda_{1} x+\lambda_{2} z, \lambda_{3} y, \lambda_{3} u\right) d \alpha d \beta d \gamma \\
& \left(\lambda_{1}=4 \alpha^{2} \beta^{2} \gamma^{2}, \lambda_{2}=4(1-\beta) \gamma(1-\gamma), \lambda_{3}=4 \alpha(1-\alpha) \beta^{2} \gamma^{2}\right), \\
& \left(\operatorname{Re}\left(a_{i}\right)>0, i=(1,2,3,4)\right) \\
& X_{25}^{(4)}\left(a_{1}, a_{1}, a_{3}, a_{1}, a_{1}, a_{2}, a_{4}, a_{2} ; c_{1}, c_{2}, c_{1}, c_{3} ; x, y, z, u\right) \\
& =\frac{\Gamma\left(c_{1}\right) \Gamma\left(c_{3}\right)}{\Gamma\left(a_{2}\right) \Gamma\left(a_{4}\right) \Gamma\left(c_{1}-a_{4}\right) \Gamma\left(c_{3}-a_{2}\right)} \\
& \times \int_{0}^{\infty} \int_{0}^{\infty} e^{-\left(a_{4} \alpha+a_{2} \beta\right)}\left(1-e^{-\alpha}\right)^{c_{1}-a_{4}-1}\left(1-e^{-\beta}\right)^{c_{3}-a_{2}-1}\left(1-z e^{-\alpha}\right)^{-a_{3}}\left(1-u e^{-\beta}\right)^{-a_{1}} \\
& \times H_{4}\left(a_{1}, 1+a_{2}-c_{3} ; c_{1}-a_{4}, c_{2} ; \frac{x\left(1-e^{-\alpha}\right)}{\left(1-u e^{-\beta}\right)^{2}},-\frac{y e^{-\beta}}{\left(1-e^{-\beta}\right)\left(1-u e^{-\beta}\right)}\right) d \alpha d \beta \\
& \left(\operatorname{Re}\left(a_{2}\right)>0, \operatorname{Re}\left(a_{4}\right)>0, \operatorname{Re}\left(c_{1}-a_{4}\right)>0, \operatorname{Re}\left(c_{3}-a_{2}\right)>0\right) .
\end{aligned}
$$

\section{Proof of the integral representations of Euler-type}

Once substituting the series definition of the special function in each integrand and then, changing the order of the integral and the summation, and finally taking into account the following integral representations of the Beta function and their various associated Eulerian integrals (see, for example, $[7,13,14,16]$ ), we derive each of the integral representations from (2.1) to (2.25). 


$$
\begin{aligned}
& B(a, b)= \begin{cases}\int_{0}^{1} t^{a-1}(1-t)^{b-1} d t, & (\operatorname{Re}(a)>0, \operatorname{Re}(b)>0), \\
\frac{\Gamma(a) \Gamma(b)}{\Gamma(a+b)}, & \left(a, b \in C \backslash Z_{0}^{-}\right),\end{cases} \\
& B(a, b)=\int_{0}^{1} \alpha^{a-1}(1-\alpha)^{b-1} d \alpha=\int_{0}^{\infty}\left(e^{-\alpha}\right)^{a}\left(1-e^{-\alpha}\right)^{b-1} d \alpha \\
& (\operatorname{Re}(a)>0, \operatorname{Re}(b)>0), \\
& B(a, b)=2 \int_{0}^{\frac{\pi}{2}}(\sin \alpha)^{2 a-1}(\cos \alpha)^{2 b-1} d \alpha=\int_{0}^{\infty} \frac{\alpha^{a-1}}{(1+\alpha)^{a+b}} d \alpha \\
& (\operatorname{Re}(a)>0, \operatorname{Re}(b)>0), \\
& B(a, b)=\frac{(S-T)^{a}(R-T)^{b}}{(S-R)^{a+b-1}} \int_{R}^{S} \frac{(\alpha-R)^{a-1}(S-\alpha)^{b-1}}{(\alpha-T)^{a+b}} d \alpha(T<R<S) \\
& =(1+M)^{a} \int_{0}^{1} \frac{\alpha^{a-1}(1-\alpha)^{b-1}}{(1+M \alpha)^{a+b}} d \alpha \quad(M>-1) \\
& (\operatorname{Re}(a)>0, \operatorname{Re}(b)>0) .
\end{aligned}
$$

\section{Integrals Representations of Laplace-Type}

Here we present certain integral representations of Laplace-Type for the functions in (1.1) to (1.5).

$$
\begin{aligned}
& X_{21}^{(4)}\left(a_{1}, a_{1}, a_{2}, a_{1}, a_{1}, a_{2}, a_{3}, a_{2} ; c_{1}, c_{1}, c_{2}, c_{3} ; x, y, z, u\right) \\
= & \frac{1}{\Gamma\left(a_{1}\right) \Gamma\left(a_{2}\right)} \int_{0}^{\infty} \int_{0}^{\infty} e^{-(s+t)} s^{a_{1}-1} t^{a_{2}-1} \\
\times & \quad\left(\operatorname{Re}\left(a_{1}\right)>0, \operatorname{Re}\left(a_{2}\right)>0\right) ; \\
& X_{22}^{(4)}\left(-; c_{1}, s_{1}^{2} x+s t y\right){ }_{1} F_{1}\left(a_{3} ; c_{2} ; t z\right)_{0} F_{1}\left(-; c_{3} ; s t u\right) d s d t, \\
= & \frac{1}{\Gamma\left(a_{1}\right) \Gamma\left(a_{2}\right)} \int_{0}^{\infty} \int_{0}^{\infty} e^{-(s+t)} a_{2} a_{1} a_{3}, a_{2} ; t_{2} a_{2}-1
\end{aligned}
$$




$$
\begin{aligned}
& \times \Psi_{2}\left(a_{2} ; c_{1}, c_{3} ; s y+t z, s u\right)_{0} F_{1}\left(-; c_{2} ; s^{2} x\right) d s d t, \\
& \left(\operatorname{Re}\left(a_{1}\right)>0, \operatorname{Re}\left(a_{2}\right)>0\right) ; \\
& X_{23}^{(4)}\left(a_{1}, a_{1}, a_{2}, a_{1}, a_{1}, a_{2}, a_{3}, a_{2} ; c_{1}, c_{1}, c_{1}, c_{2} ; x, y, z, u\right) \\
& =\frac{1}{\Gamma\left(a_{1}\right) \Gamma\left(a_{2}\right) \Gamma\left(a_{3}\right)} \int_{0}^{\infty} \int_{0}^{\infty} \int_{0}^{\infty} e^{-(s+t+v)} s^{a_{1}-1} t^{a_{2}-1} v^{a_{3}-1} \\
& \times{ }_{0} F_{1}\left(-; c_{1} ; s^{2} x+s t y+t v z\right){ }_{0} F_{1}\left(-; c_{2} ; s t u\right) d s d t d v, \\
& \left(\operatorname{Re}\left(a_{1}\right)>0, \operatorname{Re}\left(a_{2}\right)>0, \operatorname{Re}\left(a_{3}\right)>0\right) ; \\
& X_{24}^{(4)}\left(a_{1}, a_{1}, a_{2}, a_{1}, a_{1}, a_{2}, a_{3}, a_{3} ; c_{1}, c_{2}, c_{1}, c_{3} ; x, y, z, u\right) \\
& =\frac{1}{\Gamma\left(a_{1}\right) \Gamma\left(a_{2}\right) \Gamma\left(a_{3}\right)} \int_{0}^{\infty} \int_{0}^{\infty} \int_{0}^{\infty} e^{-(s+t+v)} s^{a_{1}-1} t^{a_{2}-1} v^{a_{3}-1} \\
& \times{ }_{0} F_{1}\left(-; c_{1} ; s^{2} x+t v z\right){ }_{0} F_{1}\left(-; c_{2} ; s t y\right){ }_{0} F_{1}\left(-; c_{3} ; s v u\right) d s d t d v, \\
& \left(\operatorname{Re}\left(a_{1}\right)>0, \operatorname{Re}\left(a_{2}\right)>0, \operatorname{Re}\left(a_{3}\right)>0\right) ; \\
& X_{25}^{(4)}\left(a_{1}, a_{1}, a_{3}, a_{1}, a_{1}, a_{2}, a_{4}, a_{2} ; c_{1}, c_{2}, c_{1}, c_{3} ; x, y, z, u\right) \\
& =\frac{1}{\Gamma\left(a_{1}\right)} \int_{0}^{\infty} e^{-s} s^{a_{1}-1} \\
& \times \Xi_{2}\left(a_{3}, a_{4} ; c_{1} ; z, s^{2} x\right) \Psi_{2}\left(a_{2} ; c_{2}, c_{3} ; s y, s u\right) d s,\left(\operatorname{Re}\left(a_{1}\right)>0\right) ;
\end{aligned}
$$

where $\left({ }_{0} F_{1},{ }_{1} F_{1}\right), \Psi_{2}$ and $\Xi_{2}$ denote the confluent hypergeometric functions and the Humbert functions defined, respectively, by

$$
\begin{aligned}
& { }_{0} F_{1}(-; c ; x)=\sum_{m=0}^{\infty} \frac{1}{(c)_{m}} \frac{x^{m}}{m !}, \\
& { }_{1} F_{1}(a ; c ; x)=\sum_{m=0}^{\infty} \frac{(a)_{m}}{(c)_{m}} \frac{x^{m}}{m !},
\end{aligned}
$$




$$
\Psi_{2}(a ; b, c ; x, y)=\sum_{m, n=0}^{\infty} \frac{(a)_{m+n}}{(b)_{m}(c)_{n}} \frac{x^{m}}{m !} \frac{y^{n}}{n !}
$$

and

$$
\Xi_{2}(a, b ; c ; x, y)=\sum_{m, n=0}^{\infty} \frac{(a)_{m}(b)_{m}}{(c)_{m+n}} \frac{x^{m}}{m !} \frac{y^{n}}{n !} .
$$

\section{Proof of the integral representations of Laplace-type}

It is noted that each of the integral representations (3.1) to (3.5) can be proved mainly by expressing the series definition of the involved special functions in each integrand and changing the order of the integral sign and the summation, and finally using the following well-known integral formula [1]:

$$
\Gamma(z)=\int_{0}^{\infty} e^{-t} t^{z-1} d t
$$

\section{Concluding Remarks}

Integral representations for most of the special functions of mathematical physics and applied mathematics have been investigated in the existing literature. Here we have presented some integral representations for five new quadruple hypergeometric series.

\section{References}

[1] M. G. Bin-Saad and J. A. Younis, Some integral representations for certain quadruple hypergeometric functions, Matlab J. 1 (2018), 61-68.

[2] M. G. Bin-Saad, J. A. Younis and R. Aktas, Integral representations for certain quadruple hypergeometric series, Far East J. Math. Sci. (FJMS) 103 (2018), 21-44. https://doi.org/10.17654/MS103010021

[3] M. G. Bin-Saad, J. A. Younis and R. Aktas, New quadruple hypergeometric series and their integral representations, Sarajevo Math. J. 14 (2018), 45-57.

[4] J. Choi, A. Hasanov and M Turaev, Certain integral representations of Euler type for the Exton function $X_{5}$, Honam Math. J. 32(3) (2010), 389-397. https://doi.org/10.5831/HMJ.2010.32.3.389 
[5] J. Choi, A. Hasanov and M. Turaev, Certain integral representations of Euler type for the Exton function $X_{8}$, J. Korean Soc. Math. Edu. Ser. B Pure Appl. Math. 27(2) (2012), 257264. https://doi.org/10.4134/CKMS.2012.27.2.257

[6] J. Choi, A. Hasanov, H. M. Srivastava and M. Turaev, Integral representations for Srivastava's triple hypergeometric functions, Taiwanese J. Math. 15(6) (2011), 27512762. https://doi.org/10.11650/twjm/1500406495

[7] A. Erdélyi, W. Magnus, F. Oberhettinger and F. G. Tricomi, Higher Transcendental Functions, Vol. I, New York, Toronto and London: McGraw-Hill Book Company, 1953.

[8] H. Exton, Multiple Hypergeometric Functions and Applications, New York, London, Sydney and Toronto: Halsted Press, 1976.

[9] H. Exton, Hypergeometric functions of three variables, J. Indian Acad. Math. 4 (1982), 113-119.

[10] G. Lauricella, Sulle funzioni ipergeometriche a piu variabili, Rend. Cric. Mat. Palermo 7 (1893), 111-158. https://doi.org/10.1007/BF03012437

[11] S. Saran, Hypergeometric functions of three variables, Ganita 5(2) (1954), 77-91.

[12] C. Sharma, and C. L. Parihar, Hypergeometric functions of four variables, J. Indian Acad. Math. 11 (1989), 121-133.

[13] H. M. Srivastava and J. Choi, Series Associated with the Zeta and Related Functions, Dordrecht, Boston and London: Kluwer Academic Publishers, 2001. https://doi.org/10.1007/978-94-015-9672-5_2

[14] H. M. Srivastava and J. Choi, Zeta and q-Zeta Functions and Associated Series and Integrals, Amsterdam, London and New York: Elsevier Science Publishers, 2012. https://doi.org/10.1016/B978-0-12-385218-2.00002-5

[15] H. M. Srivastava and P. W. Karlsson, Multiple Gaussian Hypergeometric Series, Bristone, London, New York and Toronto: Halsted Press, 1985.

[16] H. M. Srivastava, and H. L. Manocha, A Treatise on Generating Functions, Chichester: Ellis Horwood Ltd., 1984.

[17] J. A. Younis and M. G. Bin-Saad, Integral representations involving new hypergeometric functions of four variables, J. Frac. Calc. Appl. 10 (2019), 77-91. 